\title{
Postural adjustments associated with rapid voluntary arm movements. II. Biomechanical analysis
}

\author{
WG FRIEDLI, * L COHEN, M HALLETT, S STANHOPE, SR SIMON $\dagger$
}

From the Gait Analysis Laboratory formerly at the Rehabilitation-Engineering Center of Harvard-MIT, Department of Orthopedic Surgery, Children's Hospital Medical Center and Departments of Orthopedic Surgery and Neurology, Harvard Medical School, Boston MA, and National Institute of Neurological and Communicative Disorders and Stroke, and Biomechanics Laboratory, Department of Rehabilitation Medicine, National Institutes of Health, Bethesda MD, USA

SUMMARY Normal subjects performed bilaterally symmetric rapid elbow flexions or extensions ("focal movements") while standing. Specific patterns of electromyographic activity in leg and trunk muscles ("associated postural adjustments") were seen for each type of movement. The biomechanical significance of these postural adjustments was analysed by means of the ground reaction forces and motion of the various body segments. Experimental data were compared with that from a theoretical model of the body consisting of a six segment kinetic chain with rigid links. Distinct patterns of the ground reaction forces with elbow flexion were opposite in direction to those seen with elbow extension. Movements of the various body segments were small and specific for certain focal movement. Dynamic perturbations arising from the arm movement in an anterot posterior direction were found to be compensated by postural adjustments, whereas verticåㅇ perturbations were not compensated. The muscular activity acting about different joints in the् different movements was found to correlate with the predictions of activity needed to compensate $\frac{\mathbb{D}}{0}$ for net joint reaction moments arising from the focal movement. Motion of the various bodg segments could be understood as resulting from the interplay of the net reaction moments and the net muscular moments at the different joints. Dynamic postural requirements are accomplished b $\overrightarrow{0}$ a precise active compensation initiated before the focal movement.

Leg and trunk muscles are active in association with fast voluntary elbow movements in standing human subjects. This postural muscle activity associated with arm movements has several characteristic features. EMG activity in leg and trunk muscles precedes that of the prime mover of the displaced segment. ${ }^{12-6}$ Activity depends on the condition of postural support $^{15} 7$ including weightlessness. ${ }^{8}$ It also adapts rapidly to changes in the environmental context. ${ }^{1589}$

In our own previous study, subjects made bilaterally symmetrical rapid elbow flexions or extensions

\footnotetext{
*Present address, and address for reprint requests: Walter G. Friedli, Neurologische Universitätsklinik, Kantonsspital, CH-4031 Basel, Switzerland

†Current address: Division of Orthopedics, Ohio State University, Columbus $\mathrm{OH}$, USA.
}

Received 16 June 1987

Accepted 13 July 1987 ("focal movements") in either free standing or when supported by being strapped to a firm wall behind $\Phi$ them. EMG activity in erector spinae, rectus abdom- $\overrightarrow{\vec{B}}$ inis, quadriceps femoris, hamstrings, tibialis anterior $\frac{0}{3}$ and gastrocnemius ("postural movements") began prior to activity in the arm muscles in a distal-toproximal order of activation and demonstrated specific patterns for each type of movement. These patterns in the postural muscles were characterised by $:$ alternating activity in antagonist pairs similar to the 3 triphasic pattern seen in the arm muscles with rapid $\delta$ or "ballistic" movements. ${ }^{10-12}$ Postural "agonists" and "antagonists" were defined by this pattern 0 according to the order of activation, but the kinesiology of the postural movements was not known.

Questions arise as to the function of this muscle activity in postural muscles preceding and during $N$ voluntary arm movements. Evidence of the nature of $N$ postural strategies associated with limb displacements 
in primates ${ }^{1314}$ suggests that one goal is the minimal displacement of the centre of gravity of the animal. Posture as a state of the body is defined by two relationships, that of the body to the ground and that of the parts to each other, ${ }^{15}$ and both of which have to be preserved by postural mechanisms. The body of a normal subject standing on stable ground was found to behave as a multi-link inverted pendulum with movement not only at the ankle, but also at the knee, hip and neck. ${ }^{1617}$ As such it must be able to deal with possible forces imposed upon it. Static postural changes associated with various limb positions were described by Martin ${ }^{18}$ who proposed that adjustments were made in order to keep the centre of gravity over the feet. While passive properties of joints and muscles have been shown to be effective for postural stability during small disturbances, ${ }^{1920}$ postural strategies are supposed to require specific muscular effort with larger perturbations. Postural activity associated with voluntary arm movements in man was considered to generate anticipatory postural adjustments compensating for dynamic disturbances to balance due to the focal movement. ${ }^{245}$ In relation to a volitional arm movement these forces include not only that resulting from a new position of the limb segment but also the dynamic forces which are required to get the limb into its new position.

The analysis of the biomechanical consequences of activation of postural muscles ${ }^{1}$ during volitional (focal) arm movements is the subject of the present paper. These biomechanical effects must provide the solution to a postural problem created by the focal movement. To understand the postural problem we compare the body's kinetics and kinematics during the movement with that derived from a simulated multilink model.

\section{Methods}

The experimental procedure has been fully described elsewhere. ${ }^{1}$ Seventeen healthy male and female volunteers ranging in age from 25 to 65 years made bilaterally symmetric rapid elbow flexions or extensions ("focal movement") by moving a horizontal bar up to $90^{\circ}$ of elbow flexion, or down from $90^{\circ}$ of flexion to full extension $\left(0^{\circ}\right)$. The subjects performed these albow movements in either free standing or when supported by being strapped to a firm wall behind them (different "postural set").

Three categories of data were gathered from all the subjects participating in these experiments: the kinematics of the body including the arm movement, the ground reaction forces, and electromyograms from arm as well as from various leg and trunk muscles. Electromyographic data have been previously described in detail and will not be repeated here. ${ }^{1}$ Motions of trunk and limb segments were analysed in two subjects by the use of high-speed cinematography (Boston), in five subjects by means of a video based motion analysis system (Bethesda).

\section{Recording system}

Ground reaction forces Recordings of the foot-floor reaction forces in three orthogonal directions (vertical, anteriorposterior, medial-lateral) were made by the use of a three-dimensional force-torque dynamometer (Model OR 6-2, Amtech Inc., Newton MA). This force platform, a square $61 \mathrm{~cm}$ on each side, measured the forces and torques by a series of strain gauges arranged in a four-arm measuring bridge. The signals passed through six low noise cables to calibrated charge amplifiers. In order to get the same reference points during all trials the position of the feet on the platform was kept constant by a foot guide with an angle of $30^{\circ}$ and the heels separated by $3 \mathrm{~cm}$. The sensitivity of the platform was not affected by the foot guide.

The force plate signals were transmitted to a PDP 11-34 computer through an analog-to-digital converter, and continuously sampled every 2 milliseconds for a total of 1 second.

Body kinematics (A) High-speed cinematography (Boston): Motions of the trunk and limb segments during elbow movements were recorded by three $16 \mathrm{~mm}$ high-speed cameras, operating at $\mathbf{2 0 0}$ frames per second. These cameras were perpendicular to each other and centred at the midpoint of the force plate. For later identification of anatomical points on the film recordings, $25 \mathrm{~mm}$ squares of black adhesive tape with a white dot $10 \mathrm{~mm}$ in diameter in its centre were placed over the various anatomical sites where there was minimal skin displacement during the movements. ${ }^{2122}$ With a manual switch the cameras were started approximately 2 seconds before the onset of the photic command signal to move and were stopped after the subject's performance of the movement was visibly completed. Synchronisation of the motion data with the electrical data (force plate, electromyography) was provided by a light marker on the film simultaneous with the command signal.

(B) Video based motion analysis system (Bethesda): A video based three-dimensional motion analysis system (VICON) was used to measure kinematic and kinetic data. Eight spherical shaped markers having a diameter of $1.9 \mathrm{~cm}$ were covered with retroreflective tape and fixed to selected anatomical sites. The markers were located all on the right side of the body: in front of the ear, on the tip of the acromion ("shoulder point"), on the lateral epicondyle of the elbow, on the radial styloid at the wrist, on the anteriorsuperior iliac spine ("hip point"), on the lateral aspect of the knee ("knee point"), on the lateral malleolus of the ankle and at the base of the fifth metatarsal. Three infra-red video cameras were used to sample subject motion data. The location and orientation of each camera relative to a laboratory based coordinate system was determined using a standard Vicon calibration procedure. During the data acquisition process, the video data were sampled at $50 \mathrm{~Hz}$.

\section{Data processing}

Centre-of-pressure The projection of the centre-of-mass of the body (under static conditions) onto the horizontal plane of the force plate support with its coordinates $\mathrm{X}$ in the fore-aft and $\mathrm{Y}$ in the medial-lateral direction is defined by the centre-of-pressure. Based on the fact that the body is not rigid and has a considerable amount of inertia, the coordinates of the centre-of-pressure describe the X-Y location of $a$ the centre of gravity of the body only if no dynamic 
component is present. ${ }^{23} \mathrm{~A}$ considerable amount of ground reaction force depends upon the acceleration of the centreof-mass rather than its positional changes. ${ }^{24} 25$ With faster movements the centre-of-pressure corresponds to the position and acceleration of the centre-of-mass. The centre-ofpressure was measured by using the force plate data and its position was plotted as a function of time.

Processing of motion data High-speed cinematography data was processed as previously described. ${ }^{21}$ After being developed, the film was projected onto a Vanguard Motion Analyzer. For each of the three camera views, the twodimensional coordinates of designated anatomical points were determined on each frame using a Graf Pen digitiser that was electronically on-line to a PDP 11-34 minicomputer in which the data was then stored.

With the video based motion analysis system, the data was acquired and the two-dimensional locations of the markers were calculated by the Vicon software system.

With both methods, each point could be located to within $2.5 \mathrm{~mm}$ of its actual position. After these two-dimensional projected coordinates of each point were recorded, a subsequent program translated the coordinates into the three-dimensional coordinates used to define the locations of designated limb joints and a triangular shape representing the pelvis. From the computer-stored force plate and motion data, four-dimensional data (three spatial and one time dimension) was represented graphically by threedimensional stick figures with the superimposed foot-floor reaction force vector for each film frame. ${ }^{21}$ These figures showed the position of each segment relative to the positions of adjacent segments and foot-floor reaction force vector at each time interval and each segment's position relative to its own position at adjacent time intervals during the movement.

Three-dimensional motion data of single trials were analysed by measuring changes over time of both joint angles and anterior-posterior displacement of various anatomical points with respect to the laboratory coordinate system. These anatomical points corresponded to the "shoulder point", the "hip point" and the "knee point" in the side view. In addition, trunk motion in the sagittal plane over time was given by changes of the angle between the line connecting shoulder and hip point and the horizontal plane, that is, the floor, which we will refer to as the "trunk angle".

\section{Model}

A theoretical model of the body and the focal movement was developed in order to estimate the reaction forces the focal movement would impart on the various body parts and the ground. The model was based on Newtonian formulation and consisted of a six segment kinetic chain (the feet, shanks, thighs, trunk and head, upper arm, and forearm-hand segment). Since the focal movements were bilaterally symmetrical in nature, the bilateral anthropometrics and segment positions were modelled as single segments. Segment masses and inertia tensor data were estimated based on subject mass and height. ${ }^{26}$ The model assumed that the body was oriented vertically allowing only rotation to occur at the elbow joint. Angular displacement, velocity and acceleration of the forearm-hand segment were theoretically determined using equations 1,2 , and 3 :
(1) $\theta=\left[\left(\frac{\pi}{2}\right)\left(\frac{T_{i}}{T}-\frac{\sin \frac{2 \pi \cdot T_{i}}{T}}{2 \pi}\right)\right] \theta_{0}$

(2) $\theta=\left[\frac{\pi}{2 T}\right]\left[\sin \left(\frac{2 \pi T_{i}}{T}-\frac{\pi}{2}\right)+1\right]$

(3) $\theta=\left(\frac{\pi}{T}\right)^{2}\left(\sin \frac{2 \pi T_{i}}{T}\right)$

where: $\pi=3 \cdot 141592654$

$T_{i}=$ instantaneous time value

$\mathrm{T}=$ total movement time

$\theta_{0}=$ value of theta when $T_{i}=0$

Phi was defined as the superior angle formed by the forearmhand segment and the vertical $(\mathrm{Z})$ laboratory axis. Through the use of inverse dynamic analysis, joint reaction forces and moments acting on each model segment were calculated utilising the kinematics of the forearm-hand segment.

\section{Results}

\section{Ground reaction forces}

In order to understand how the ground reaction $\vec{\sim}$ forces were related in time to the event of the i symmetrical arm movement and to the forces gene? $\omega_{N}$ ated by the postural muscles, the force plate data were 을 qualitatively analysed in the vertical and anterioß posterior direction. The forces in the medio-laterid T⿱ direction had small amplitudes and presumablos 음 represented only the degree of asymmetry of the movements.

Ground reaction forces were found to be fair $\vec{Q}$ consistent in successive trials with each condition $\infty$ within the same subject. Therefore, average force plate data was used for comparison between different conditions (fig 1).

For elbow flexions performed in the unsupported state the vertical reaction forces were characterised by a distinct sinusoidal single cycle pattern (fig la). There was an initial force reaching a maximum amplitude $\frac{1}{3}$ greater than body weight approximately $\frac{1}{3}$ of the time into the arm movement. This was followed by a reversal in the force direction, yielding minimum values $\frac{1}{3}$ below body weight late during the arm movement. These main features were present in all subjects. Variability was seen in the transition from the maximum to the minimum force from subject to subject; rather than having the appearance of a smooth sinusoidal oscillation, small deviations or "bumps" were occasionally visible in this portion of the signal. After having reached a minimal value (approximately $\frac{2}{3}$ body weight) the signal again showed a reversal in its direction with a second peak greater than body weight of small amplitude occurring after the movement was over. This was then followed by a number of sinusoidal oscillations of 


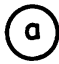

Flexion

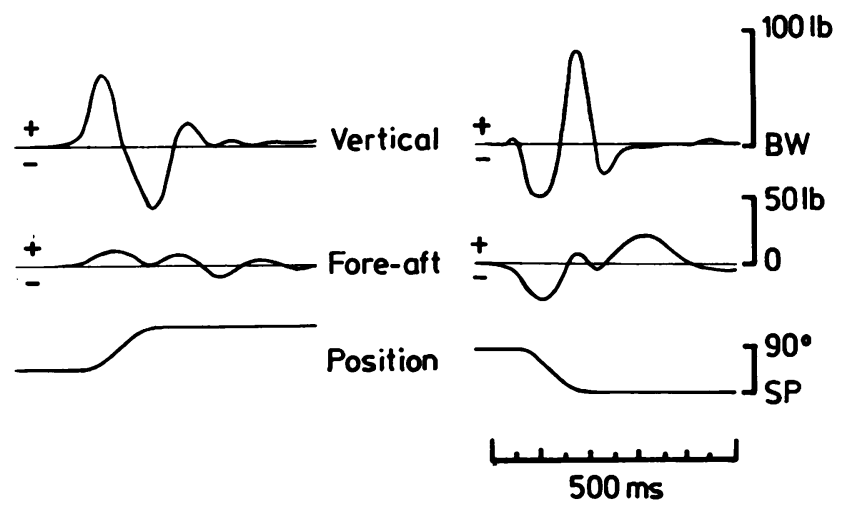

(b) Supported

Flexion

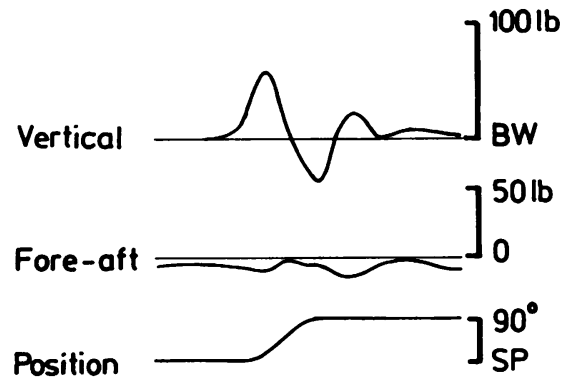

Fig 1 Position records (as measured by the potentiometer) and ground reaction forces. Data for elbow flexion are compared with that for extension in the unsupported state $(a)$ as well as elbow flexion with body support (b). Positive sign represents vertical force in addition to body weight $(B W)$, or forward-directed force in the antero-posterior direction. Average force and position data of 10 elbow movements per condition from one subject.

very small amplitude. The ground reaction forces in the antero-posterior direction were small in amplitude. Starting at the onset of the arm movement there was a forward directed force (positive sign). Its peak was reached slightly before the time of the first maximum force noted in the vertical direction. The forward directed reaction force turned to zero or reached a small maximum backward force coincident with the vertical force minimum. There then followed a number of small sinusoidal oscillations.

Comparison of the supported with the unsupported condition revealed no significant difference in the phasic pattern of the vertical component of the reaction force (fig $1 b$ ). In all subjects slightly smaller amplitudes of both maximum and minimum force values were seen with body support. The pattern of forces in the for-aft direction was opposite to that seen in the unsupported state and the amplitude was slightly less. Peak reaction forces were backward directed. The baseline itself was shifted in the backward direction which was probably due to the subjects' leaning against the wall.

The sinusoidal oscillation pattern of both the vertical and antero-posterior components with elbow extension was opposite to that seen with elbow flexion in the unsupported state (fig la). While the initial minimum of the vertical force was smaller in magnitude than the initial force maximum with elbow flexion, the positive peak in the second half of the movement had maximum values of up to $40 \%$ greater than body weight. A clear backward reaction force maximum was seen shortly after the first minimum was reached in the vertical direction, followed by a small forward peak coincident with the positive maximum of the vertical component.

\section{Centre-of-pressure}

Since the arm movements performed were bilaterally symmetrical, positional changes of the centre-ofpressure in the antero-posterior direction had more relevance than the minor medial-lateral changes again representing presumably minor asymmetries of the focal movement.

With elbow flexion (fig 2A) the centre-of-pressure in the antero-posterior direction moved backwards for

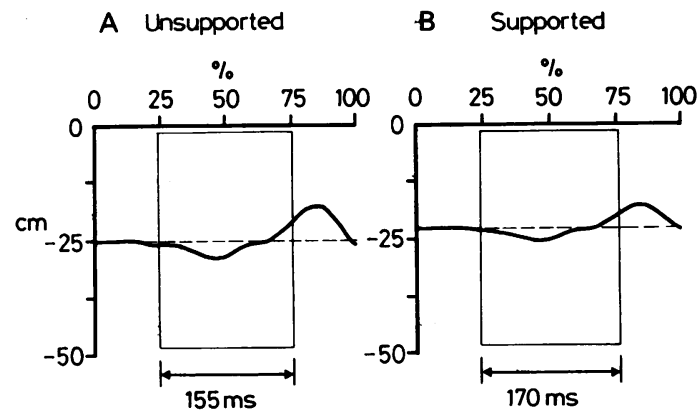

Fig 2 Displacement of the centre of pressure over time with elbow flexion in the unsupported $(A)$ and supported (B) state. Backward shift is down, forward shift is up. Time scale is given by percentages of the sum total of digitised frames ( 62 in $A, 68$ in $B ; 5 \mathrm{~ms}$ per frame). The boxes indicate the duration of elbow movement in $A$ and $B$. 


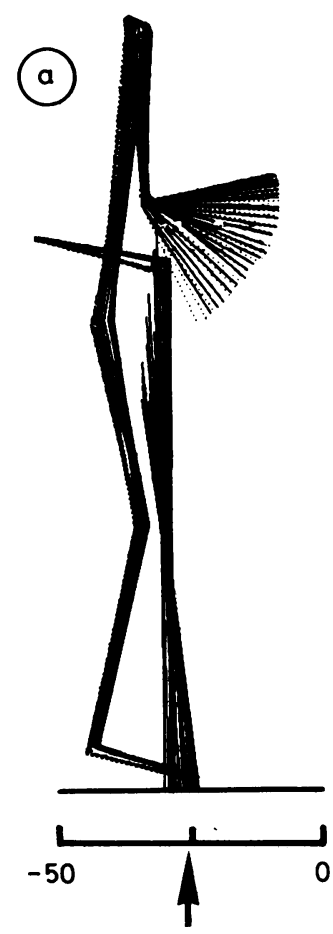

Initial position of $\mathrm{CP}$

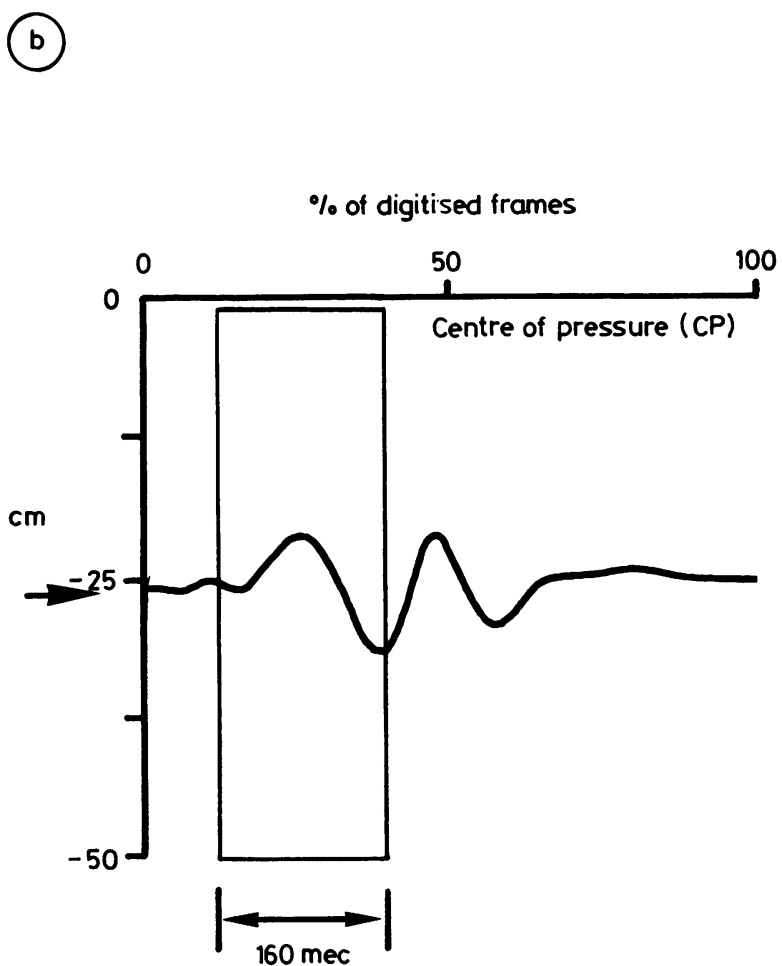

Fig 3 Positional changes of the centre of pressure over time with elbow extension. Superimposed stick figures and ground reaction force vectors are shown for the whole duration of arm movement (160 ms). The arrow indicates the initial positionof the centre of pressure on the floor in the sagittal plane (a). Displacement of the centre of pressure in the antero-posteriof direction over time is shown with respect to the elbow movement as marked by the box (b). Forward is up, backward motion of the centre of pressure on the floor is down. Time scale is given as in fig 2 (120 frames digitised; 5 ms per frame). Representative data from one subject.

the first half of elbow movement, reaching a maximum of less than $5 \mathrm{~cm}$ with respect to its starting position. During the second half of the elbow movement the centre-of-pressure returned to the starting position and then shifted forward to a maximum displacement of approximately $8 \mathrm{~cm}$ before coming back to the initial position after the elbow flexion had ended.

With elbow flexion in the supported state (fig 2B) changes in the centre-of-pressure were qualitatively similar as without body support. While characterised by a backward displacement during the arm movement and a forward shift after the latter had ended, both maxima were of less magnitude compared with that seen without body support. Moreover the initial position of the centre-of-pressure was shifted forward in the supported state.

With elbow extension (fig 3) the directional sinusoidal cycle pattern covered by the centre-of- pressure over time was reverse of that seen with flexion movements. There was a forward displacement of the centre-of-pressure reaching a maximum of about $5 \mathrm{~cm}$ with respect to the starting position in the middle of the arm movement. This was followed by a backward shift providing a backward maximum coincident with the end of elbow extension. There was another oscillation before the centre-of-pressure got back to its initial position after the focal movement had ended.

\section{Motion data}

Because of the bilateral symmetry, the motion of the various body segments was analysed in the sagittal plane. The analysis focused on body motions which occurred early since these are most directly related to the biomechanical events resulting in part from the early activation of postural muscles.

The onset of arm movement as defined by the first 
sign of wrist marker displacement was 20 to $45 \mathrm{~ms}$ earlier than that measured by the potentiometer which was used for the earlier analysis of EMG data. ${ }^{1}$ In this paper the analysis of both dynamics of the focal movement and its timing relative to motion of the various body segments is based entirely on the motion data.

The extent of elbow movements was approximately $80^{\circ}$ and the duration ranged from 160 to $180 \mathrm{~ms}$ with peak angular velocities of up to $800^{\circ}$ per second for the forearm segment. Although subjects were instructed to hold the upper arm vertical and adjacent to the trunk during the elbow movement, shoulder motion did occur and was in the range of $5^{\circ}$ to $8^{\circ}$.

In general, motions of trunk and lower body segments associated with fast voluntary elbow movements was small in magnitude (fig 4) reaching their peak about half way into the focal movement (fig 5). Angular displacements included hip flexion of about $2.5^{\circ}$, an increase of the angle between the shank and the foot of about $2^{\circ}$ with hardly any angular displacement at the knee. Accordingly, small positional changes were evident in the antero-posterior direction for hip, knee and shoulder points. There was backward shift of the hip by about $1.2 \mathrm{~cm}$ simultaneously with that of the knee by about $0.8 \mathrm{~cm}$ whereas forward displacement of the shoulder was in the range of 0.6 $\mathrm{cm}$. A decrease of the trunk angle by about $2^{\circ}$ was a consequence of the forward trunk flexion as indicated by positional changes described above. However, this trunk flexion seen initially for a short period of time changed to trunk extension in accordance with clear backward shift of the shoulder by up to $2.0 \mathrm{~cm}$ starting early during the focal movement. Later on, about half way into the focal movement, positional changes took place in lower limb segments opposite in direction to those appearing with the movement onset. These were characterised by a forward displacement of hip and knee and increasing hip and knee extension. A more detailed description of these body motions occurring in the second half and after cessation of the focal movement will be given for elbow extension where these changes were opposite in direction but of larger extent compared with those with elbow flexinn.

Angular changes taking place during the first half of elbow extension were of small magnitude (fig 5). There was a decrease of the angle between the shank and the foot by about $2^{\circ}$. Minor angular deflection representing knee extension of less than $2^{\circ}$ was seen in some of the subjects while in the others changes of the knee joint angle were not measurable initially during the elbow movement. An increase of hip flexion by some $2 \cdot 5^{\circ}$ was measured along with a decrease of the trunk angle by about $2^{\circ}$. Positional changes of anatomical points over time illustrated kinematics of the body segments associated with elbow extension (fig 6).

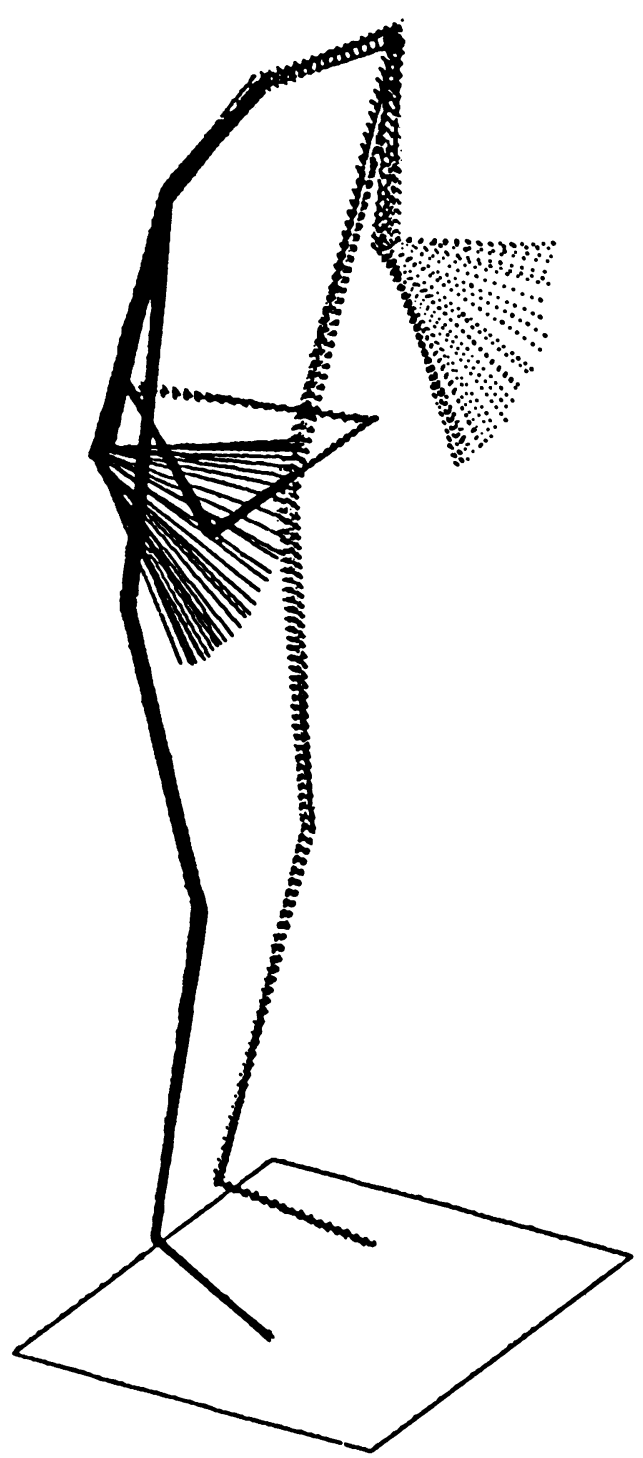

Fig 4 Elbow flexion in the unsupported state. Threedimensional stick figures as calculated from two-dimensional coordinates of designated anatomical points are superimposed for the whole duration of arm movement (160 ms). Note minimal motion of trunk and lower body segments as well as of the upper arm.

There was forward displacement of both knee and hip by 0.7 to $0.8 \mathrm{~cm}$ starting with the focal movement and being accomplished about half way into it. There then followed a reversal in direction with a backward shift of knee and hip by some 10 to $15 \mathrm{~mm}$ beyond their initial position. This cycle of fore-aft displacement 

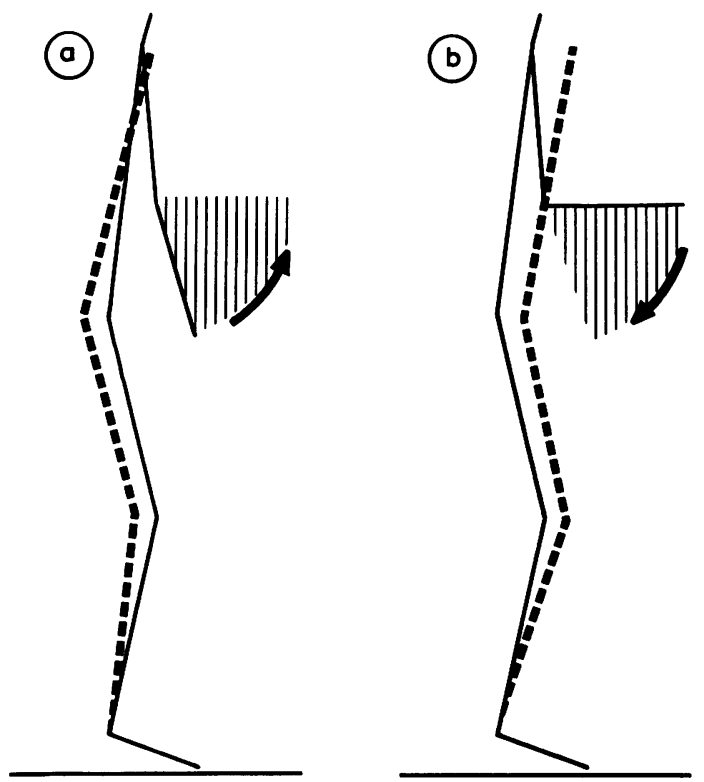

Fig 5 Schematic diagrams of postural adjustments as seen initially during elbow flexion and extension. The direction of the focal movement is given by the arrow, the range is indicated by the hatched area. Body motion based on calculated kinematic data is delineated excessively for better visualisation. While the solid stick figures represent posture before the onset of the focal movement, early changes of the body segments with respect to each other are shown by dotted stick figures.

was accompanied by continuous forward shift of the shoulder by an average of $2.5 \mathrm{~cm}$. According to these changes later during the elbow movement there was evidence for hip and knee flexion as well as forward bending of the trunk. Maximum values were reached 100 to $150 \mathrm{~ms}$ after the focal movement had ended, followed by changes in the opposite direction (fig 7). However, the final relationship between the various body segments was slightly different from that seen before the initiation of elbow movement.

\section{Calculated parameters}

Based on our model the two main components of the reactive forces imparted on the body by the focal movement were calculated for a six segment kinetic chain with rotation only at the elbow joint: first, the translational reaction forces and, second, the joint reaction moments resulting from the torque at the elbow.

Translational reaction forces An attempt to understand the force plate data (representing the total of forces arising from the focal movement as well as postural activity) was to compare the theoretical translational reaction forces contributed by the moving arm with the real ground reaction forces. Comparison revealed strong resemblances between the calculated values and experimental measurements for the sinusoidal oscillation pattern of the vertical component, reaching a force maximum in the first half and minimum values late during the elbow movement (fig 8). In contrast to the good agreement of both timing and magnitude of the vertical component, significant differences were found between the calculated forces and that measured by the force plate for the force component in antero-posterior direction (fig 8). The calculations provided a biphasic oscillation pattern with a forward force maximum approximately $\frac{1}{5}$ of the time into the focal movement, followed by a reversal in the force direction, yielding a pronounced
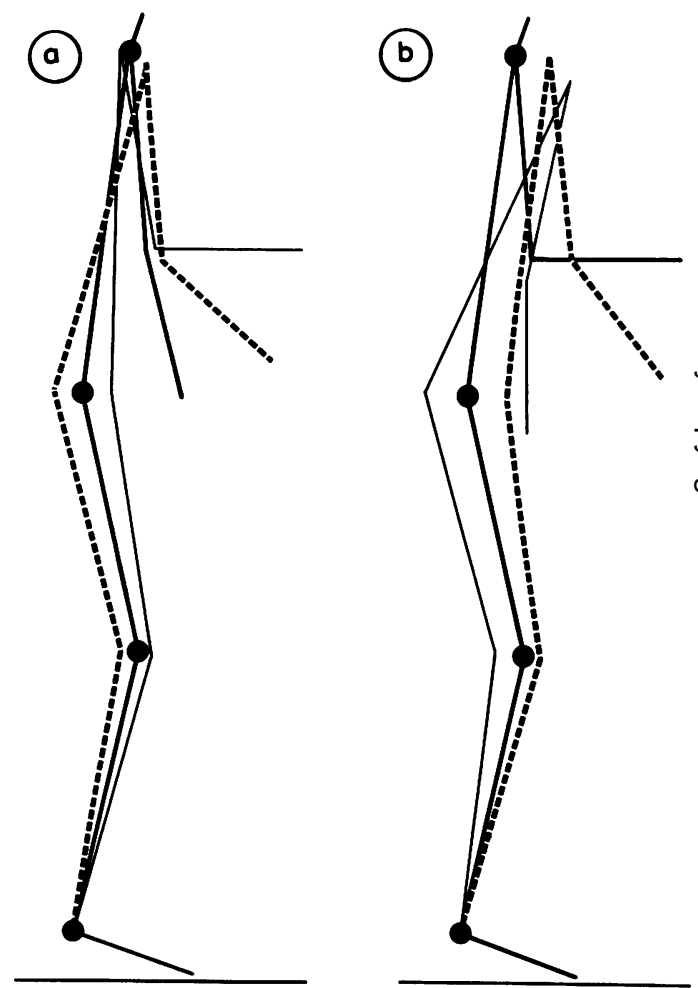

Fig 6 Schematic diagrams of sequential stick figures representing body motion associated with elbow flexion (a) and extension (b) over time. The initial positions of shoulder, hip and knee are indicated by the dots. The broken stick figures represent positional changes due to the displacement of knee, hip and shoulder initially during the elbow movement and being accomplished about half way into it. Further positional changes as represented by the third stick figure were opposite in direction reaching peak amplitudes after cessation of the focal movement. See text for further details. 
Right hip flexion

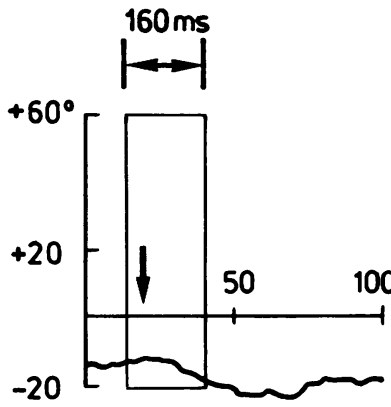

Right knee flexion

$160 \mathrm{~ms}$
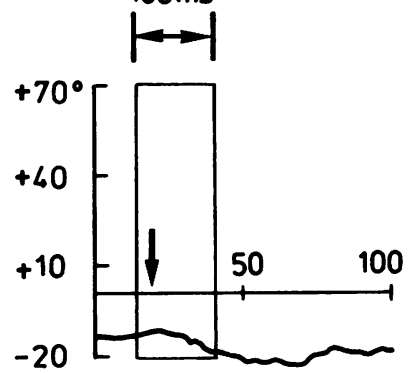

Right foot flexion

$160 \mathrm{~ms}$

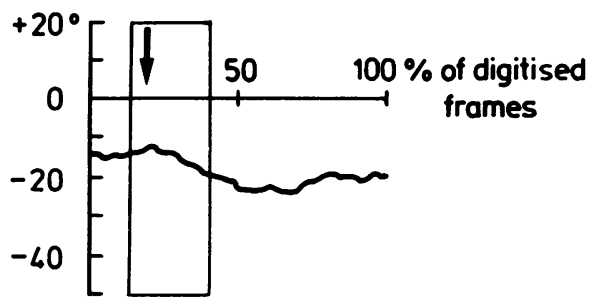

Fig 7 Joint angles in the right leg over time as calculated from digitised film data for an elbow extension. Angles are calculated with respect to the vertical ( = zero degrees). Relative extension is up, relative flexion is down. Time scale is given by the percentage of the sum total of digitised frames (120;5 ms per frame). The box indicates the focal movement (duration $160 \mathrm{~ms}$ ). Small changes initially during the elbow movement (arrows) were followed by major changes opposite in direction and overlasting the focal movement. Note that the joint angles did not reach their original values at the end.

backward maximum during the deceleration phase of the arm movement. In contrast to these changes based on theoretical calculations, the real antero-posterior forces were of small magnitude, reaching peak amplitudes of less than $\frac{1}{10}$ of the calculated values. While the two curves were in phase initially during the focal movement, the real ground reaction forces did not

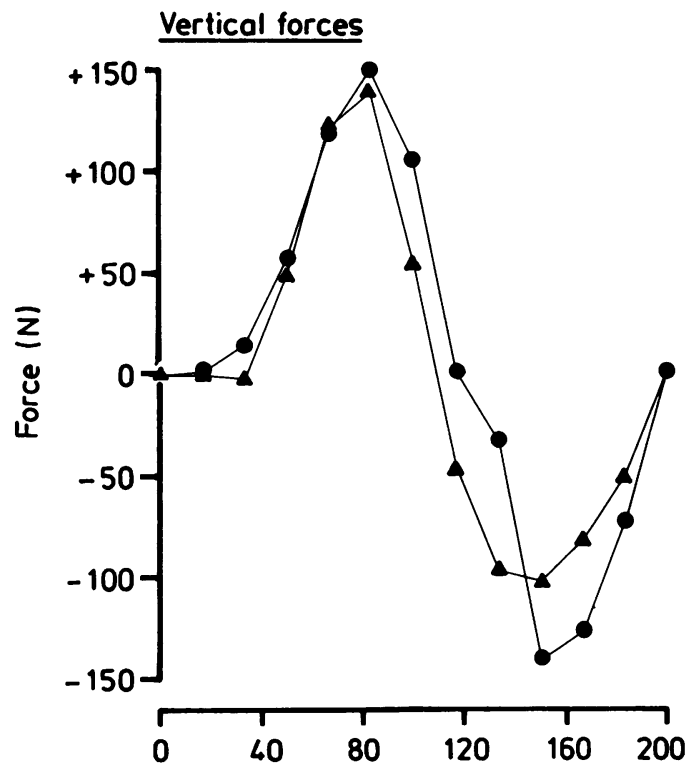

show a backward maximum in the second half of the arm movement and were missing a biphasic sinusoidal pattern.

Joint reaction moments The "net joint reaction moments" which describe the total influence on the joint as a result of the focal movement were calculated by utilising the kinematics of the forearm-hand segment. These moments reflect components arising

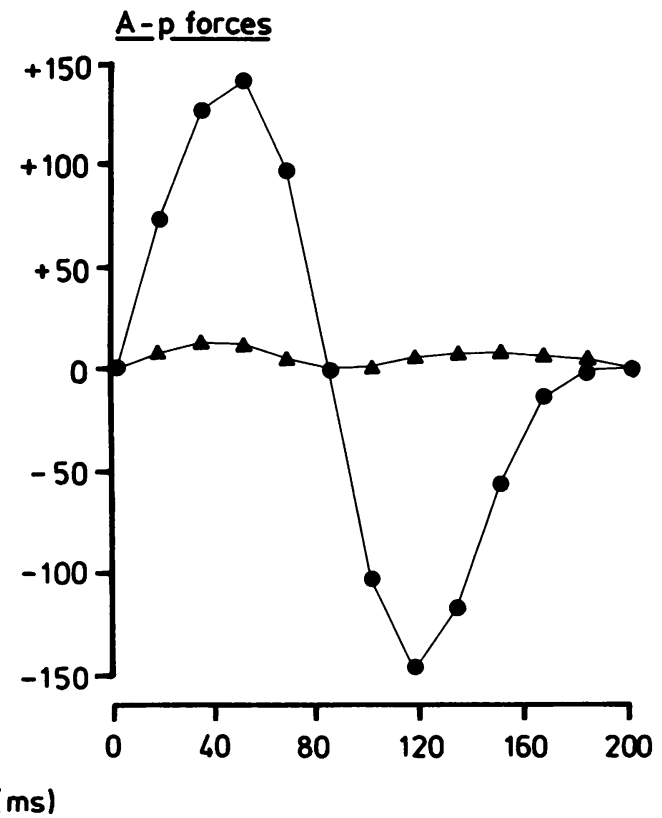

Fig 8 Comparison between calculated ground reaction forces (O) and experimental force data as measured by the force plate $(\mathbf{\Delta})$ in the vertical and antero-posterior direction for an elbow flexion movement (duration $200 \mathrm{~ms}$ ). Positive sign indicates vertical force in addition to body weight, or forward-directed force in antero-posterior direction. 

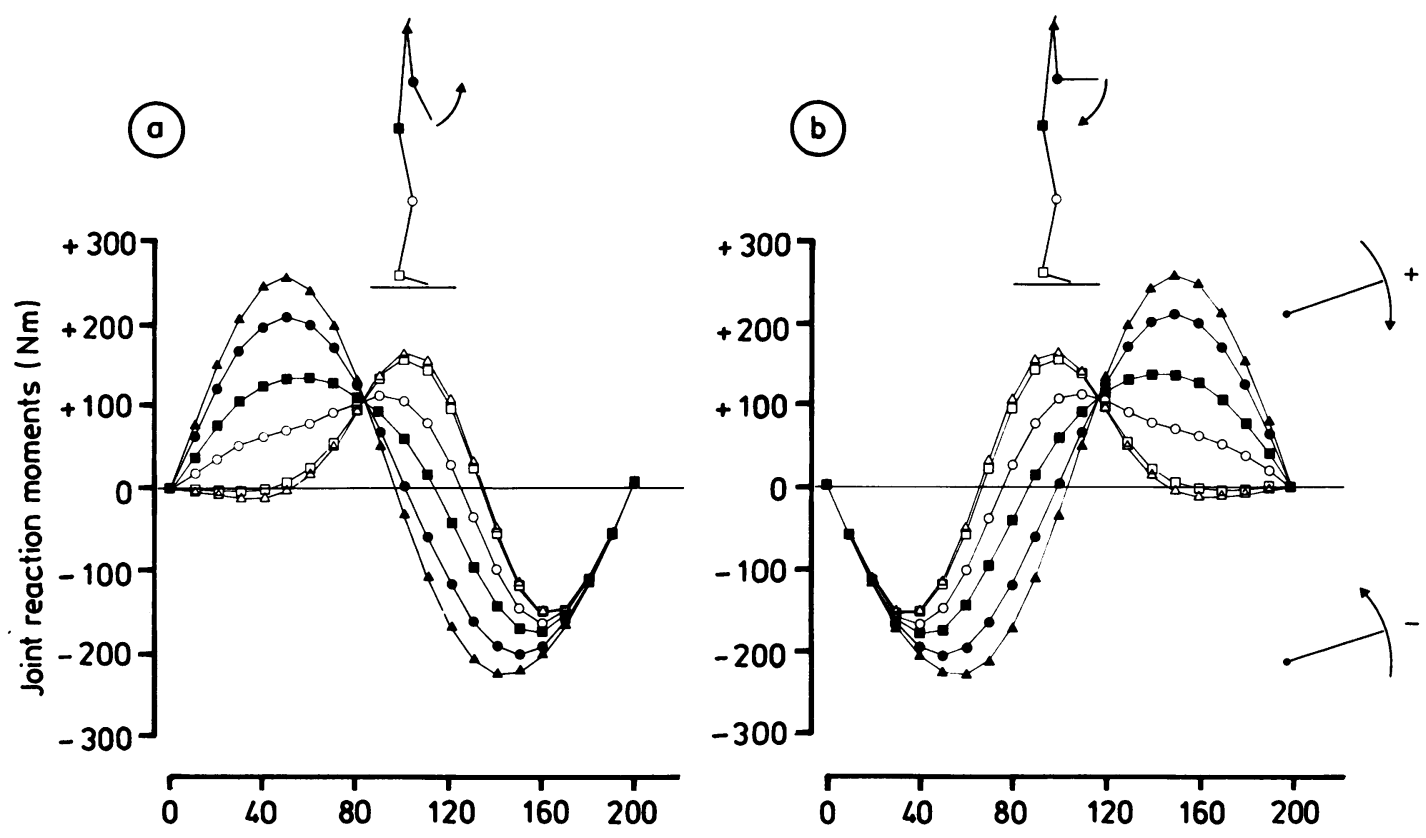

Time (ms)

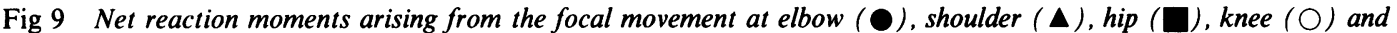
ankle $(\square)$ as well as at the base of support $(\triangle)$. Changes over time are given as theoretically calculated for the whole duration of elbow flexion (a) and extension (b). Clockwise moments are positive, counterclockwise moments are negative In $a$, the rotational and translational components are in the same direction for the upper extremities but in the opposite direction for the lower extremities. Thus, the moments for the upper extremities peak before those in the legs. In $b$, the opposite is true: the translational component acts in the same direction as the rotational component in the legs, and therefore the moments peak sooner.

both from rotation and the crossproduct of translational force times distance. For each type of elbow movement the net reaction moments at each joint were plotted through the entire movement (fig 9).

For the basic flexion movement (fig 9a) the initial net joint reaction moments were clockwise (positive values) at hip and knee, reaching peak values of up to $120 \mathrm{Nm}$ which were followed by a reversal in direction with negative maxima late during the focal movement. As an exception to the general pattern of joint reaction moments there was negligible transient counterclockwise moment at the ankle after the onset of the focal movement; this then turned into distinct clockwise moment with a maximum in the order of magnitude of $130 \mathrm{Nm}$ after peak values had occurred at the hip and before showing another negative oscillation with a counterclockwise maximum during the last fourth of the elbow movement. With elbow extension (fig $9 \mathrm{~b}$ ) the pattern of net reaction moments at the various joints over time was the reverse of that seen with elbow flexion, that is, characterised by a sinusoidal oscillation pattern but with counter- clockwise moments at hip, knee and ankle and negative maxima during the first half of the focal moment.

\section{Discussion}

The postural problem

For postural stability with voluntary arm movement while standing the body has to deal with the dynamic forces imparted to it by the focal movement. These forces are coupled to the trunk at the shoulder and will act on the multisegmental kinematic chain between the shoulder and the base of support. These forces include both translational and rotational components. In addition, a displacement of the body centre-of-mass with respect to the ground is expected to arise from the focal movement. Therefore, if stability is to be maintained during voluntary arm movement, several requirements have to be met by associated postural adjustments: First, compensation for dynamic perturbation produced by the focal movement and, second, static balance at the endpoint of the movement. Postural adjustments have to 
counterbalance all the forces tending to move the centre-of-gravity beyond the base of support ${ }^{2728}$ in order to prevent the body from falling. In addition, the body angles have to be maintained within limits in order to preserve a relatively constant relationship of the various body segments to each other.

A theoretical model of the body has been used to define the problem for the body set up by the focal movement. Given that very little movement of the body is observed in association with the focal movement, the assumption of the body as a six segment kinetic chain with rigid links seems reasonable as the first approximation. The model provides information about the two components of the reactive forces on the body due to the displacement of the forearm segment. While the translational component is part of the ground reaction forces as measured by the force plate, the model allows a theoretical evaluation of the portion of the reaction forces contributed by the moving arm. The joint reaction moments as the second component of the reactive forces on the body result from the torque at the elbow arising from the arm movement. The "net joint reaction moments" provided by our model describe the total influence on the various joints as a result of the focal movement.

\section{Interpretation of data}

Distinct patterns of the ground reaction forces with elbow flexion are opposite in direction to those seen with elbow extension (fig 1) and therefore are considered to be specified by the dynamics of the focal movement. Comparison between the ground reaction forces and the translational reaction forces arising from the arm movement as given by the theoretical kinetic analysis provides information as to the postural strategy of the body. While the theoretically evaluated forces are in good agreement with the experimental measurements for the vertical component, they are found to differ significantly for the antero-posterior component. The anteroposterior reaction forces measured by the force plate are of much smaller magnitude and do not show a biphasic sinusoidal pattern with a distinct backward maximum later during the focal movement (fig 8). The latter was expected to occur with deceleration of the moving segment as represented by the anteroposterior component of the translational reaction forces due to the arm movement. Based on these findings, the vertical component of the ground reaction forces mainly represents the reaction to forces generated by the moving arm. On the other hand, the antero-posterior component does not correspond to the translational reaction force and must be strongly influenced by forces generated by other muscular activity. Hence, compensation for dynamic perturbation of the body in antero-posterior direction is suggested by the small amplitude of the ground reaction forces. This compensation presumably helps to keep the centre-of-gravity over the base of support and may be provided by only small movements of the body since the mass of the body is much larger than that of the forearm segment. This interpretation is supported by the findings when the body was supported by the wall and unable to move in the antero-posterior direction. The vertical force measured by the platform is only slightly changed (fig 1). External antero-posterior forces are exerted by the wall as a replacement for postural muscle activity which is significantly diminished in this condition. The pattern of the measured forces differs, and their amplitude remains low.

Depending upon the acceleration of the body centre-of-mass, positional changes of the centre-ofpressure over time represent a complex measure although they suggest some directional specificity with respect to the focal movement. Only before the initiation and after the termination of postural adjustments associated with the focal movement, that is, when no considerable dynamic component is present, does the location of the centre-of-pressure correspond to the projection of the centre-of-mass perpendicular to the floor. The centre of pressure returns close to its starting position at the end of the movement although the position of the forearm-hand segment has changed. This finding indicates that the net movement of the centre-of-mass has been minimal. This has been accomplished by only small movements of the body segments.

Although the various body segments are found to move very little, our results suggest directional specificity of the body movements according to the dynamics of the forthcoming focal movement. Motion of the body segments with respect to each other results from both forces imparted to the body by the moving arm and forces generated by the postural muscles. The former are given by the net joint reaction moments arising from the arm movement while the effect of the latter might be qualitatively estimated on the basis of postural adjustments which demonstrate specific EMG patterns for each type of movement. ${ }^{1}$ Comparison between the net joint reaction moments and the muscular moments as expected to arise from specific postural patterns provides information as to the strategy of the body in maintaining the relationship of the body segments with respect to each other. According to our previous results ${ }^{1}$ the muscular forces generated by postural agonists are antagonistic to the net joint reaction moments occurring initially during the focal movement. For example, the initial net joint reaction moments with elbow flexion are clockwise at hip and knee (positive sign in fig 9) and are compensated for by the early activation 
of erector spinae, hamstrings and gastrocnemius producing moments opposite in direction to those arising from the arm movement. A small transient counterclockwise moment initially during elbow flexion preceding the marked clockwise moment at the ankle represents an exception to the general pattern seen for other movements. This small downward shift of the curve is due to the large antero-posterior translational reaction force generated at the onset of the movement. A marked clockwise reaction moment at the shoulder in the first half of elbow flexion is opposed by the early activation of anterior deltoid as the postural agonist (fig 10 of ref 1 ) which provides fixation of the upper arm to the trunk. Both the net joint reaction moments and body motion with elbow flexion are opposite in direction to those seen with elbow extension which is in accordance with the postural pattern being reverse for the two focal movements. Hence, the body movements which are seen reflect the interplay of forces (net joint reaction moments and muscular forces) at each joint.

The fact that the resultant movement is small represents the main finding of our analysis. Moments about each joint created by the focal arm movement should lead to large body movements. The body's postural reaction is very effective in minimising these effects and producing stability. In general, the postural muscle activity is stronger than the net joint reaction moment so that the small movements are in the direction specified by the postural muscle. An exception is the initial flexion of the hip as the arm begins to flex. Under the dominant influence of the net joint reaction moment (first causing a rotational shift of the trunk) it might be useful to allow a slight flexion of the hip rather than to maintain a rigid link, since hip flexion will "absorb" some of the reactive force so that it does not propagate to more distal links while simultaneously maintaining the centre-of-gravity within the base of support. The relative delay of hip extension (fig 5) therefore is not contradictory to activation of the paraspinal muscles and may result from the clockwise moment at the hip due to the focal movement. Phasic activity of both gastrocnemius and tibialis anterior is found to be weak compared to that seen in thigh- and trunk muscles ${ }^{1}$ which presumably indicates the major role of hip stabilisation with rapid arm movements while standing. ${ }^{27}$

The body motions during the latter part of the arm movement are not related to the biomechanical events resulting from postural muscle activity anticipating the prime mover onset and are probably influenced by sensory feedback control. With both elbow flexion and extension the initial changes are followed by some antiphase motion of trunk and legs which outlast the focal movement and have the aspect of damped oscillations (fig 6). Body motion opposite in direction to that seen initially during the elbow movement might be expected to occur according to the reversal in direction of the net joint reaction moments in the second half of the focal movement (fig 9). Additionally, the postural muscles exhibit alternating burst patterns. Comparison between the net joint reaction moments over time and the timing of postural muscles suggests some compensatory effect not only of the postural agonists but also of the antagonists. Both might be properly timed in order to counteract the sequential changes of the net joint reaction moments arising from the focal movement.

\section{Conclusions}

Postural adjustments have to counterbalance translational and rotational forces arising from the focal movement in order to accomplish the postural requirements of preventing the body from falling and maintaining the relationship of the various body segments to each other.

According to our results, dynamic perturbations arising from the arm movement in antero-posterior direction are compensated by postural adjustments whereas vertical perturbations are not compensated Muscular activity acting about different joints is found to correlate to the predictions of activity needeo to compensate for the net joint reaction moments due to the focal movement at the different joints. Motion of the various body segments which results from the interplay of the net joint reaction moments and the muscular moments is small, and represents an optimak solution of the postural problem.

The normal mechanism of postural control is shown to be a precise compensation provided by the activation of postural muscles. Postural activity anticipating the arm movement indicates that the body prefers to deal with postural stabilisation in a feedforward rather than in a feedback mode. Both timing and magnitude of appropriate postural muscles are considered to be critical with respect to the precision of "postural movements". According to former results ${ }^{30-32}$ the examination of patients with cerebellar and basal ganglia disease may therefore provide further information on the role played by these systems for postural stabilisation.

Walter G. Friedli was supported by a grant from the Schweizerische Stiftung für Medizinisch-Biologische Stipendien. The work was supported by grants from NIHR (23-P-55854/1), United Cerebral Palsy Research and The Charles A. Dana Foundation Inc.

\section{References}

1 Friedli WG, Hallett M, Simon SR. Postural adjustments associated with rapid voluntary arm movements. I. Electro- 
myographic data. J Neurol Neurosurg Psychiatry 1984; 47:611-22.

2 Belenkii VY, Gurfinkel VS, Paltsev YL. Elements of control of voluntary movements. Biofizika 1967;12:154-61.

3 Paltsev YL, Elner AM. Preparatory and compensatory period during voluntary movement in patients with involvement of the brain of different location. Biofizika 1967;12:161-8.

4 Bouisset S, Zattara M. A sequence of postural movements precedes voluntary movement. Neurosci Lett 1981;22:263-70.

5 Cordo PJ, Nashner LM. Properties of postural adjustments associated with rapid arm movements. J Neurophysiol 1982; 47:287-302.

6 Lee WA. Anticipatory control of postural and task muscles during rapid arm flexion. $J$ Motor Behav 1980;12:185-96.

7 Marsden CD, Merton PA, Morton HB. Human postural responses. Brain 1981;104:513-34.

8 Clément G, Gurfinkel VS, Lestienne F. Mechanisms of posture maintenance in weightlessness. In: Igarashi M, Black FO, eds. Vestibular and Visual Control on Posture and Locomotor Equilibrium. Basel: Karger, 1985: 158-63.

9 Gurfinkel VS, Kots YM, Paltsev EI, Feldman AG. The compensation of respiratory disturbances of the erect posture of man as an example of the organization of interarticular interaction. In: Gelfand IM, Gurfinkel VS, Fomin SV, Tselin ML, eds. Models of the Structural-functional Organization of Certain Biological Systems. Cambridge: MIT Press, 1961: 382-95.

10 Angel RW. Myoelectric patterns associated with ballistic movements: effects of unexpected changes in load. Human Movement Studies 1975;1:96-103.

11 Freund $\mathrm{HJ}$. Büdingen $\mathrm{HJ}$. The relationship between speed and amplitude of the fastest voluntary contractions of human arm muscles. Exp Brain Res 1978;31:1-12.

12 Hallett M, Marsden CD. Ballistic flexion movements of the human thumb. J Physiol (London) 1979;294:33-50.

13 Gahery Y, Nieouillon A. Postural and kinetic coordination following cortical stimuli which induce flexion movements in the cat's limbs. Brain Res 1978;149:25-38:

14 Massion J. Role of motor cortex in postural adjustments associated with movement. In: Asanuma $\mathbf{H}$, Wilson V, eds. Integration in the Nervous System. Tokyo-New York: Igaku-Shoin, 1979: 239-60.

15 Martin JP. A short essay on posture and movement. $J$ Neurol
Neurosurg Psychiatry 1977;40:25-9.

16 Roberts TDM, Stenhouse G. The nature of postural sway. Agressologie 1976;17A:11-4.

17 Camana PC, Hemami H, Stockwell CW. Determination of feedback for human posture control without physical intervention. J Cybern 1977;7:199-225.

18 Martin JP. The Basal Ganglia and Posture. London: Pitman Medical, 1967.

19 Gurfinkel VS, Lipshits MI, Popov KY. Is the stretch reflex the main mechanism in the system of regulation of the vertical posture of man? Biophysics 1974;19:744-8.

20 Nashner LM. Adapting reflexes controlling the human posture. Exp Brain Res 1976;26:59-72.

21 Simon SR, Nuzzo RM, Koskinen MF. A comprehensive clinical system for four-dimensional motion analysis. Bull Hosp Joint Dis 1977;38:41-4.

22 Simon SR, Deutsch SD, Nuzzo RM, et al. Genu recurvatum in spastic cerebral palsy. J Bone Joint Surg 1978;60A:882-94.

23 Kodde L, Geursen JB, Venema EP, Massen C. A critique on stabilograms. J Biomed Eng 1979;1:123-4.

24 Thomas DP, Whitney RJ. Postural movements during normal standing in man. $J$ Anat (London) 1959;93:524-39.

25 Gurfinkel VS. Physical foundations of stabilography. Agressologie 1973;14C:9-14.

26 Dempster WT. Space requirements of the seated operator. Wright Patterson Air Force Base, Dayton OH, Rep WADCTR 1955: 55-159.

27 Murray MP, Seireg A, Scholz RC. Center of gravity, center of pressure, and supportive forces during human activities. $J$ Appl Physiol 1967;23:831-8.

28 Murray MP, Seireg A, Sepic SB. Normal postural stability and steadyness: quantitative assessment. J Bone Joint Surg 1975;57A:510-6.

29 Nashner LM. Adaptation of human movement to altered environments. Trends Neurosci 1982;5:358-61.

30 Hallett $M$, Khosbin S. A physiological mechanism of bradykinesia. Brain 1980;103:301-14.

31 Grimm RJ, Nashner LM. Long loop dyscontrol. In: Desmedt JE, ed. Prog Clin Neurophysiol, vol 4. Basel: Karger, 1978: 70-84.

32 Traub MM, Rothwell JC, Marsden CD. Anticipatory postural reflexes in Parkinson's disease and other akinetic-rigid syndromes and in cerebellar ataxia. Brain 1980;103:393-412. 